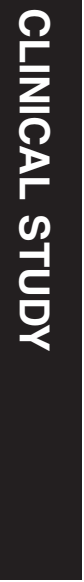

\title{
Outcomes of microcatheter-assisted trabeculotomy following failed angle surgeries in primary congenital glaucoma
}

${ }^{1}$ Beijing Tongren Eye Center, Department of Ophthalmology, Beijing Tongren Hospital, Capital Medical University, Beijing, China

${ }^{2}$ Department of Ophthalmology,

Massachusetts Eye and Ear Infirmary, Boston, MA USA

${ }^{3}$ Department of Ophthalmology, Beijing Anzhen Hospital, Beijing, China

${ }^{4}$ Department of Ophthalmology, First People's Hospital of Xuzhou, Xuzhou, China

Correspondence: N Wang, Beijing Tongren Eye Center, Department of Ophthalmology, Beijing Tongren Hospital, Capital Medical University,

1 Dongjiaominxiang Street, Dongcheng District, Beijing 100730, China

Tel: +86105826 9920;

Fax: +861058 269920

E-mail: wningli@vip.163.

com

Received: 11 March 2016 Accepted in revised form: 31 August 2016 Published online: 14 October 2016

\begin{abstract}
Purpose To report surgical outcomes of microcatheter-assisted trabeculotomy following failed angle surgeries, and compare those with no previous angle surgery, in primary congenital glaucoma (PCG). Methods The early postoperative (12 months) results of 42 eyes of 36 patients who underwent microcatheter-assisted trabeculotomy by single surgeon for PCG were retrospectively analyzed. Group 1, 20 eyes of 16 patients, had no previous angle surgery. Group 2, 22 eyes of 20 patients, had one or two previous failed angle surgeries. Success was defined as an intraocular pressure (IOP) $<21 \mathrm{~mm} \mathrm{Hg}$ with at least a $30 \%$ reduction from preoperative IOP with (qualified success) or without (complete success) the use of antiglaucoma medication. Results Mean IOP decreased from $31.5 \pm 7.2 \mathrm{~mm} \mathrm{Hg}$ on 3 (median, range: $1-5$ ) medications in Group 1 and $34.6 \pm 7.3 \mathrm{~mm} \mathrm{Hg}$ on 3 (median, range: 1-4) medications in Group 2 preoperatively to $15.6 \pm 3.1 \mathrm{~mm} \mathrm{Hg}$ on 0 (median, range: $0-4$ ) medications in Group 1 and $16.0 \pm 4.6 \mathrm{~mm} \mathrm{Hg}$ on 0 (median, range: $0-2)$ medications in Group 2 postoperatively at 12 months (both $P<0.001$ ), respectively. The mean percentage of IOP reduction from preoperative to last postoperative visit was $46.0 \pm \mathbf{2 0 . 1} \%$ in Group 1 and $45.5 \pm 25.0 \%$ in Group $2, P=0.947$.

Qualified and complete successes were comparable between Group 1 and Group 2 (qualified success: $\mathbf{9 0 . 0} \%$ vs $\mathbf{7 7 . 3} \%, P=0.294$; complete success: $78.9 \%$ vs $77.3 \%, P=0.853$ ). Complications were minimal. Conclusions Microcatheter-assisted trabeculotomy achieved significant pressurelowering effects with a reduction in medication use in PCG, and it represents a
\end{abstract}

Y Shi ${ }^{1}$, H Wang ${ }^{1}$, J Yin ${ }^{2}$, X Zhang ${ }^{1}$, M Li ${ }^{1}$, C Xin ${ }^{3}$, $X$ Chen $^{4}$ and N Wang ${ }^{1}$ reasonable choice of initial and repeat surgical treatment for PCG.

Eye (2017) 31, 132-139; doi:10.1038/eye.2016.212; published online 14 October 2016

\section{Introduction}

Trabeculotomy ab externo is the mainstay and initial procedure of choice for the treatment of primary congenital glaucoma (PCG) ${ }^{1-4}$ particularly, in children with corneal opacity that prevents adequate visualization of the angle. Rigid probe trabeculotomy has an overall success of $\sim 60-87 \%$ after a mean follow-up of 1-3 years. ${ }^{5-8}$ Additional trabeculotomies, however, are often needed in eyes with uncontrolled intraocular pressure (IOP) owing to its inability to open the full circumference of the Schlemm's canal with a single incision. ${ }^{9,10}$

Previous angle surgeries make subsequent angle surgeries more challenging, as intact area reserved for additional surgery is limited. Beck and Lynch $^{11}$ first described using 6-0 polypropylene to circumvent the entire $360^{\circ}$ of the trabecular meshwork in patients with PCG. Nevertheless, threading a suture around the Schlemm's canal entails a risk of misdirection into the suprachoroidal space $\mathrm{e}^{12,13}$ and it may be even more difficult to advance the suture around the full circumference in eyes with previous angle surgeries. ${ }^{14}$ Recently, it has been reported that a complete or partial trabeculotomy was safely completed in eyes with childhood glaucoma with the assistance of an illuminated microcatheter, and that it provided significant IOP-lowering effects. ${ }^{15,16}$ To date, the feasibility and effect of microcatheter-assisted trabeculotomy in eyes with previous failed angle surgeries have not been reported to our knowledge. In this study, we report surgical 
outcomes of microcatheter-assisted trabeculotomy in patients with PCG following failed angle surgeries, compared with those with no previous angle surgery.

\section{Subjects and methods}

\section{Study design}

Our preliminary results on an interim analysis of the first 20 patients suggested PCG patients without previous surgery demonstrated a $90.0 \%$ qualified success rate after receiving the microcatheter-assisted trabeculotomy. In our clinic experiences, for those with previous sugeries, the qualified success rate of traditional rigid probe trabeculotomy was only 50\%. We estimated an increase of $20 \%$ in terms of the qualified success for this novel procedure to treat PCG patients with previous surgeries. With a two-sided test using a level of 0.05 and a power of $80 \%$, the sample size was 18 subjects per arm. On the basis of the above data, clinical data of consecutive case series of eyes undergoing trabeculotomy by single surgeon using an ophthalmic microcatheter (iTRACK 250A; iScience Interventional, Menlo Park, CA, USA) for PCG were collected from January 2014 to May 2015 at Beijing Tongren Eye Center. This retrospective case series study was approved by the Ethics Committee of Beijing Tongren Eye Center and adhered to the tenets of the Declaration of Helsinki, and it was registered under the Chinese Clinical Trials Registry (ChiCTR-OCC-15005789). Each patient's legal guardian or representative signed an informed consent. All patients had complete eye examination with detailed history and were diagnosed with PCG with the following characteristics including isolated trabeculodysgenesis without any other ocular or systemic abnormalities, elevated IOP, increased corneal diameter, corneal edema, increased axial length, and glaucomatous cupping of the optic nerve. IOP was measured at baseline and on follow-up examinations using the Icare tonometer (Icare TA01i, Icare Finland Oy, Espoo, Finland) while the subject was asleep. Patients without previous angle surgeries comprise Group 1 and those who underwent no more than two previous failed angle surgeries (trabeculotomy, trabeculotomy combined with trabeculectomy or goniotomy) comprise Group 2. Patients were further stratified into neonatal PCG (with onset after birth and $<1$ month), infantile PCG (with onset after 1 month and $<2$ years of age), and late-onset PCG (with onset after 2 years of age). ${ }^{17}$ Exclusion criteria included patients who previously had more than two angle surgeries in the candidate eye or underwent other ophthalmic surgery besides aforementioned angle surgeries. An initial examination under anesthesia was performed using an $80 \mathrm{MHz}$ ultrasound biomicroscopy (iUltrasound, iScience Interventional) in all cases to determine suitability for trabeculotomy surgery. Cases with $>180^{\circ}$ of peripheral anterior synechiae were excluded. If the patients' eyes met the criteria for inclusion, both eyes were included in the analysis.

\section{Surgical procedure and postoperative care}

The details have been described previously. ${ }^{15,16}$ Briefly, following a conjunctival peritomy, the Schlemm's canal was exposed by either scleral cut down under a superficial scleral flap or direct unroofing via a deep scleral flap. In those with previous surgeries, the site of sclera flap was located laterally to previous flap (or bleb if existed) for at least $60^{\circ}$ to avoid the possible damage of Schlemm's canal in previous surgeries. The two ostia of the canal were dilated with high-molecular weight hyaluronic acid (Healon GV, Abbott Vision, Abbott Park, IL, USA), similarly to a viscocanalostomy. Once the Schlemm's canal was entered, the illuminated microcatheter was inserted into the canal and threaded circumferentially with the illuminated tip providing precise location of the catheter. In cases of successful $360^{\circ}$ catheterization, the catheter tip will be retrieved from the other ostium, and both exposed ends of the catheter were grasped and pulled in opposite directions like a purse string, thus breaking through the trabecular meshwork and performing the trabeculotomy ab externo. In some cases, the microcatheter would reach an obstruction or become misdirected. In these cases, the conjunctiva was incised and a scleral cut down was performed over the illuminated catheter tip, which was retrieved at the point of obstruction. Both exposed ends of the catheter were then grasped and pulled in opposite directions like a purse string performing a partial trabeculotomy. In all cases, the scleral flap was then closed with interrupted 10-0 nylon sutures and the conjunctiva was approximated with 8-0 vicryl sutures. Gentle anterior chamber irrigation was performed via a paracentesis for rare occasions of significant hyphema. Postoperatively, tobramycin/ dexamethasone (TobraDex, Alcon, Rijksweg, Belgium) 4 times per day were used initially (tapering over 2-4 weeks), along with Panoprofen (Pranopulin, Senju Pharmaceutical Co., Ltd, Osaka, Japan) 4 times daily for $\sim 4$ weeks and pilocarpine $2 \%$ (Bausch \& Lomb, Rochester, NY, USA) solution 3 times daily for 1 to 3 months. We routinely use pilocarpine for at least 1 month to prevent the development of peripheral anterior synechiae after trabeculotomy regardless of the IOP and it was not considered as an IOP-lowering medication during this time. If the IOP was higher than $21 \mathrm{~mm} \mathrm{Hg}$ after 1 month, the pilocarpine was continued as an IOP-lowering medication. To prevent the side effects of the long-term use of pilocarpine, the length of treatment did not exceed 3 months. 


\section{Outcome measures and statistical analysis}

Clinical charts and operative reports were reviewed and the following information was recorded: demographics, duration of follow-up, age at the time of surgery, onset of the disease, preoperative diagnosis and prior surgeries, horizontal corneal diameter, the extent of successful trabeculotomy (degree), interoperative and postoperative complications, IOP, and medications at each follow-up visit. All patients were followed up at 1, 3, 6, 9, and 12 months post operation. Demographic and clinical characteristics between the groups were compared using the Student's $t$-test and $\chi^{2}$-test. Repeated-measures analysis of variance with Bonferroni adjustment was used to compare IOP and medications from preoperative baseline to 12 months between two groups. The median number of medication reduction, follow-up duration, and degree of angle with successful trabeculotomy were compared between two groups using the nonparametric Mann-Whitney $U$-test.

Success was defined as an IOP $<21 \mathrm{~mm} \mathrm{Hg}$ with at least a $30 \%$ reduction from preoperative pressure levels with (qualified success) or without (complete success) the use of antiglaucoma medication. Kaplan-Meier survival functions were constructed for complete and qualified success, and compared between groups using a robust variance estimator to account for the clustering of eyes within patients. Eyes undergoing a secondary surgical procedure were defined as failure and excluded from analysis subsequent to the intervention. The mean percentage of IOP reduction from preoperative to last postoperative visit (ie, at the last follow-up visit or when failure criteria were reached) were compared between groups using a mixed statistical model to account for the clustering of eyes within a given patient, and were compared between eyes with complete $360^{\circ}$

trabeculotomy and partial trabeculotomy using the Mann-Whitney $U$-test. Correlation analysis was carried out by Spearman correlation test. A $P$-value of $<0.05$ (two-sided) was considered significant. All statistical tests were performed using the software SPSS 16.0 (SPSS, Inc., Chicago, IL, USA).

\section{Results}

\section{Demographics and preoperative parameters}

We enrolled 20 eyes of 16 patients without previous angle surgeries (Group 1) and 22 eyes of 20 patients with previous failed angle surgeries (Group 2). All 42 eyes of 36 patients were followed for a minimum of 6 months and the median follow-up duration was 12 (range: 6-12) months in both groups. No statistical differences were found with respect to gender $(P=0.095)$, horizontal corneal diameter $(P=0.907)$, cup to disc ratio $(P=0.680)$, number of eyes with cloudy cornea $(P=0.569)$, and follow-up duration $(P=0.513)$ between the two study groups (Table 1). Disk assessment at baseline was only available on 25 eyes owing to corneal clouding. It is worth noting that the average age at the time of surgery was older in Group 2 (48.5 \pm 29.8 , range: $4-108$ months) than in Group 1 (33.5 \pm 32.2 , range: 3-96 months), although the difference was not statistically significant $(P=0.125)$. In Group 2, 16 eyes $(72.7 \%$ ) had 1 previous surgery (5 eyes with trabeculotomy, 11 eyes with trabeculotomy combined with trabeculectomy) and 6 eyes (27.3\%) had 2 previous surgeries (either trabeculotomy or trabeculotomy combined with trabeculectomy). No bleb was presented in any of our patients.

\section{Degree of trabeculotomy with either microcatheter or trabeculotome}

Schlemm's canal was cannulated with the catheter, either completely or partially, in all cases. More eyes in Group 1 (15 eyes, $75.0 \%$ ) than in Group 2 (4 eyes, 18.2\%) had complete $360^{\circ}$ trabeculotomy. More eyes in Group 2 (18 eyes, $81.8 \%$ ) than in Group 1 (5 eyes, 25.0\%) had partial trabeculotomy with microcatheter. Among the five eyes in Group 1, three were neonatal, one was infantile, and one was late-onset PCG. Among the 18 eyes in Group 2, six were neonatal PCG and three of them had two previous surgeries, 10 were infantile PCG, and three of them had two previous surgeries, and two were late-onset PCG with one previous surgery. The median degree of successful trabeculotomy was significantly higher in Group 1 (360, range: 120-360) than in Group 2 (195, range: 120-360, $P=0.007)$.

\section{Change in IOP and antiglaucoma medication usage}

Mean IOP decreased from $31.5 \pm 7.2 \mathrm{~mm} \mathrm{Hg}$ in Group 1 and $34.6 \pm 7.3 \mathrm{~mm} \mathrm{Hg}$ in Group 2 preoperatively to $15.6 \pm 3.1 \mathrm{~mm} \mathrm{Hg}(P<0.001)$ and $16.0 \pm 4.6 \mathrm{~mm} \mathrm{Hg}$ $(P<0.001)$ postoperatively at 12 months, respectively. Median number of medications decreased from 3 (range: 1-5) in Group 1 and 3 (range: 1-4) in Group 2 preoperatively to 0 (range: $0-4 ; P=0.002$ ) in Group 1 and 0 (range: $0-2 ; P<0.001$ ) in Group 2 postoperatively at 12 months. In both groups, IOP and medication use were significantly reduced from baseline at all postoperative visits (both $P<0.001$, repeated-measures analysis). Multivariable repeated-measures analysis of variance controlling for age $(P=0.571)$, age of onset $(P=0.963)$, horizontal corneal diameter $(P=0.258)$, and preoperative IOP $(P=0.530)$ was performed to adjust for possible confounding factors or imbalances, as well as the influences of these 4 covariates on IOP. This revealed no significant differences in IOP and the mean number of 
Table 1 Demographic and preoperative parameters of children with childhood glaucoma having microcatheter-assisted trabeculotomy

\begin{tabular}{|c|c|c|c|c|}
\hline Parameter & Total & $\begin{array}{c}\text { Cases without } \\
\text { previous surgery } \\
\text { history }\end{array}$ & $\begin{array}{c}\text { Cases with } \\
\text { previous surgery } \\
\text { history }\end{array}$ & $\mathrm{P}$ \\
\hline Patients & 36 & 16 & 20 & \\
\hline Number of eyes & 42 & 20 & 22 & $0.758^{\mathrm{a}}$ \\
\hline Average age, months, mean \pm SD (range) & $41.4 \pm 31.5(3-108)$ & $33.5 \pm 32.2(3-96)$ & $48.5 \pm 29.8(4-108)$ & $0.125^{\mathrm{b}}$ \\
\hline \multicolumn{5}{|l|}{ Sex, number of patients } \\
\hline Male & 24 & 13 & 11 & $0.095^{\mathrm{c}}$ \\
\hline Female & 12 & 3 & 9 & \\
\hline \multicolumn{5}{|l|}{ Diagnosis by eye, $\mathrm{n}$} \\
\hline Neonatal PCG (onset: birth-1 month) & 9 & 3 & 6 & $0.553^{\mathrm{a}}$ \\
\hline Infantile PCG (onset: 1 month-2 years) & 24 & 13 & 11 & \\
\hline Late-onset PCG (onset: > $>2$ years) & 9 & 4 & 5 & \\
\hline Horizontal corneal diameter, $\mathrm{mm}$, mean $\pm \mathrm{SD}$ & $12.8 \pm 1.1$ & $12.8 \pm 1.1$ & $12.8 \pm 1.1$ & $0.907^{\mathrm{b}}$ \\
\hline Cup to disc ratio & $0.78 \pm 0.12$ & $0.79 \pm 0.06$ & $0.77 \pm 0.15$ & $0.680^{\mathrm{b}}$ \\
\hline Cloudy cornea, $n(\%)$ & $25(59.5)$ & $11(55.0)$ & $14(63.6)$ & $0.569^{\mathrm{a}}$ \\
\hline Follow-up duration, months, median (range) & $12(6-12)$ & $12(6-12)$ & $12(6-12)$ & $0.513^{\mathrm{d}}$ \\
\hline Average previous surgeries, mean $\pm S D$ & - & - & $1.3 \pm 0.5$ & - \\
\hline $1 n(\%)$ & & & $16(72.7)$ & \\
\hline $2 n(\%)$ & & & $6(27.3)$ & \\
\hline Preoperative IOP, mean \pm SD & $33.1 \pm 7.4$ & $31.5 \pm 7.2$ & $34.6 \pm 7.3$ & $0.171^{\mathrm{b}}$ \\
\hline $\begin{array}{l}\text { Preoperative number of medications, mean } \pm \mathrm{SD} \text { (median, } \\
\text { range) }\end{array}$ & $2.7 \pm 0.9(3,1-4)$ & $2.7 \pm 1.1(3,1-5)$ & $2.7 \pm 0.8(3,1-4)$ & $0.958^{\mathrm{d}}$ \\
\hline
\end{tabular}

Abbreviation: $n$, number of eyes.

${ }^{\mathrm{a}} \chi^{2}$-test.

' Independent-samples $t$-test.

'Fisher's exact test.

dMann-Whitney U-test.

medications between the two groups at any postoperative time point $(P>0.05$, Figure 1$)$. The mean percentage of IOP reduction from preoperative to last postoperative visit was similar between both groups $(46.0 \pm 20.1 \%$ in Group 1 and $45.5 \pm 25.0 \%$ in Group 2; $P=0.947)$. The median number of medications decreased from 3 (range: 1-5) in Group 1 and 3 (1-4) in Group 2 preoperatively to 0 (range: $0-4$ ) at last postoperative visit in both groups with no difference between the two groups $(P=0.792)$.

The mean percentage of IOP reduction was not significantly different between eyes with complete $360^{\circ}$ trabeculotomy $(46.0 \pm 20.1 \%$, mean \pm SD) and partial trabeculotomy with microcatheter $(45.5 \pm 25.0 \%$, mean $\pm \mathrm{SD}, P=0.947$ ), and it had a positive correlation with the extent of trabeculotomy, but this was not statistically significant $(r=0.179, P=0.256$; Spearman rank correlation).

\section{Success rate}

Table 2 shows the cumulative success rate at various postoperative time points and Figure 2 shows the
Kaplan-Meier survival plots. At 12-month follow-up, Group 1 achieved a 78.9\% complete and $90.0 \%$ qualified success rate, whereas Group 2 achieved 77.3\% and 77.3\%, respectively. There was no statistical difference between the two groups (complete success: $P=0.853$, Figure 2, left; qualified success: $P=0.294$, Figure 2, right). In Group 2, one eye underwent transscleral diode laser cyclophotocoagulation at 9-month follow-up, hence was considered a failure and censored from analysis subsequent to the intervention. Even in those eyes with partial trabeculotomy with microcatheter, 4 out of 5 eyes in Group 1 and 13 out of 18 eyes in Group 2 had successful control of IOP $(<21 \mathrm{~mm} \mathrm{Hg}$ with or without medications) with mean IOP reduction of $45.6 \pm 20.1 \%$ in Group 1 and $55.1 \pm 12.1 \%$ in Group 2 at last follow-up.

\section{Complications and secondary procedures}

Blood reflux was noted intraoperatively and minimal to small hyphema was seen on the first postoperative day in all subjects. Two subjects in Group 2 had small amounts of residual blood in the anterior chamber at their 1-week 


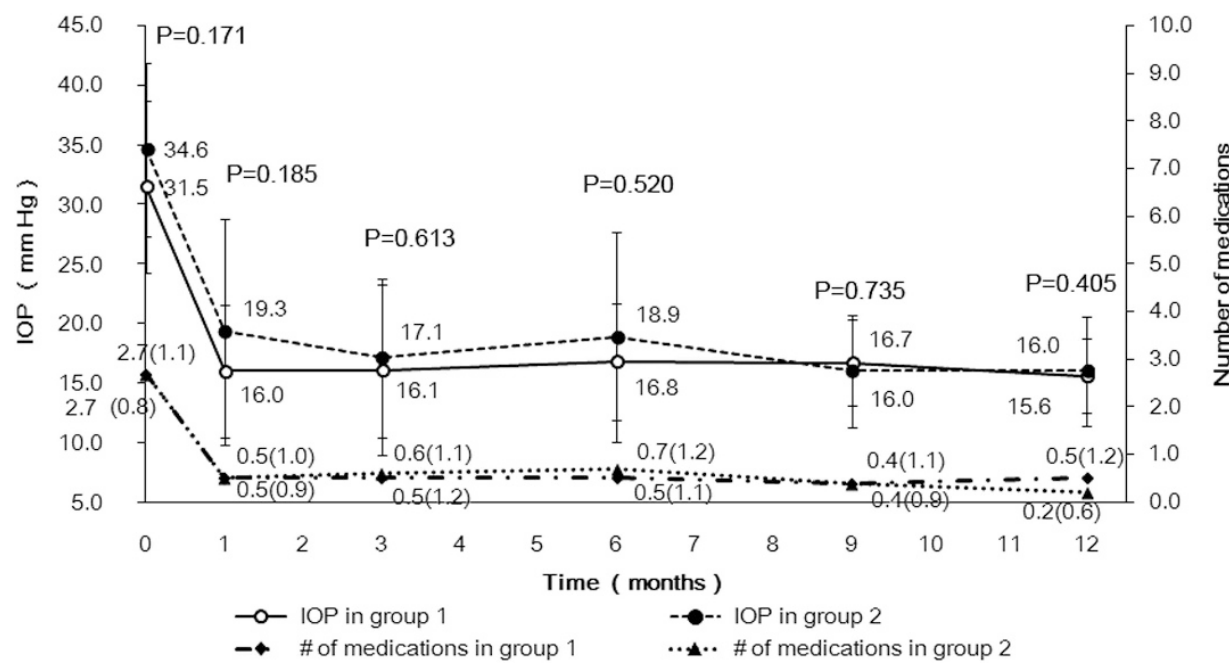

Figure 1 Comparison of mean IOP and number of medications between eyes without previous angle surgery (Group 1) and eyes with previous angle surgeries (Group 2) after microcatheter-assisted trabeculotomy for pediatric glaucoma over 12-month follow-up. No significant differences were found between the two groups at any time point. Number of medications: mean (SD).

Table 2 The cumulative success rate of microcatheter-assisted trabeculotomy in childhood glaucoma

\begin{tabular}{|c|c|c|c|c|c|c|}
\hline \multirow[t]{2}{*}{ Follow-up } & \multicolumn{2}{|c|}{ Complete success (\%) } & \multicolumn{2}{|c|}{ Qualified success (\%) } & \multicolumn{2}{|c|}{$\mathrm{N}$ (eyes) } \\
\hline & Group 1 & Group 2 & Group 1 & Group 2 & Group 1 & Group 2 \\
\hline 1 month & 85.0 & 86.4 & 90.0 & 84.2 & 20 & 22 \\
\hline 3 months & 85.0 & 81.8 & 90.0 & 90.9 & 20 & 22 \\
\hline 6 months & 85.0 & 77.3 & 90.0 & 86.4 & 20 & 22 \\
\hline 9 months & 78.9 & 77.3 & 90.0 & 77.3 & 16 & 21 \\
\hline 12 months & 78.9 & 77.3 & 90.0 & 77.3 & 14 & 17 \\
\hline
\end{tabular}

Group 1: eyes without previous angle surgery; Group 2: eyes with previous angle surgeries. Success was defined as an intraocular pressure (IOP) $<21 \mathrm{~mm}$ $\mathrm{Hg}$ with at least a 30\% reduction from preoperative pressure levels with (qualified success) or without (complete success) the use of antiglaucoma medication. Eyes undergoing a secondary surgical procedure were classified as failures and censored from analysis subsequent to the intervention.

postoperative assessment. At the 1-month follow-up examination, no patient in either group had any evident hyphema. No cases of iris tear, Descemet's tears, choroidal detachment, or persistent hypotony were noted.

\section{Discussion}

Despite the reported high success rate (80-90\%) of trabeculotomy in PCG, Ikeda et al ${ }^{9}$ reported that to achieve successful control of IOP, 59.8\% eyes required 1 trabeculotomy, $18.8 \%$ required 2 trabeculotomies, and $7.1 \%$ required 3 trabeculotomies, and some even required trabeculectomy $(2.7 \%)$ or cyclocryotherapy $(0.9 \%)$ in addition to the trabeculotomies. Given the relatively high success rate and low incidence of intra and postoperative complications of trabeculotomy, as compared with mitomycin C-augmented trabeculectomy, aqueous shunts, and cyclodestructive surgery, 8,10 two or three trabeculotomies may be performed in eyes with uncontrolled IOPs in our practice, similar to what was reported by others. ${ }^{9,18-20}$ Although these cases are typically considered to have poor prognosis, the previous angle surgeries may make these cases even more challenging, owing to the limited area intact for additional surgery and difficulty accessing the inferior region with the trabeculotome. The main advantage of microcatheter-assisted trabeculotomy is the ability to treat the entire angle to the maximum degree with a single incision, especially the inferior angle for eyes with previous angle surgeries, thereby maximizing IOPlowering benefit. In this retrospective study, a significant reduction of IOP and medication use was detected in PCG with previous failed angle surgeries treated by microcatheter-assisted trabeculotomy. Although the degree of Schlemm's canal canalized by the microcatheter was less in these eyes than that in eyes undergoing this procedure as an initial intervention owing to previous surgical destruction of Schlemm's canal, the IOP-lowing 

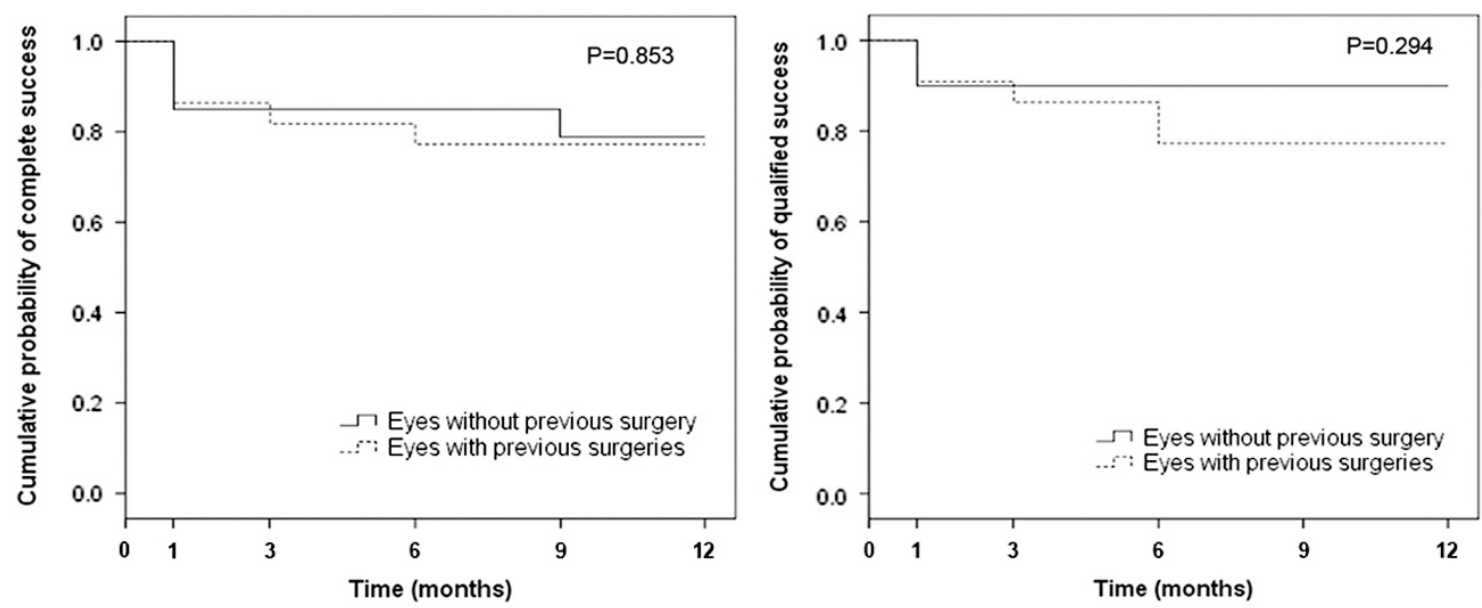

Figure 2 Kaplan-Meier survival plot of the cumulative probability of success for eyes without previous angle surgery (Group 1) and eyes with previous angle surgeries (Group 2) after microcatheter-assisted trabeculotomy for pediatric glaucoma over 12 months. Success was defined as an IOP $<21 \mathrm{~mm} \mathrm{Hg}$ with at least a $30 \%$ reduction from preoperative pressure levels with (qualified success) or without (complete success) the use of antiglaucoma medication. Eyes undergoing a secondary surgical procedure were classified as failures and censored from analysis subsequent to the intervention. The differences between groups were analyzed by the log-rank test.

effect was comparable between these two groups $(77.3 \%$ vs $90.0 \%$ qualified success rate, $77.3 \%$ vs $78.9 \%$ complete success rate for eye with $v s$ without previous angle surgery). The mean percentage of IOP reduction was not significantly different between eyes with complete $360^{\circ}$ trabeculotomy $(46.0 \pm 20.1 \%$, mean $\pm S D)$ and partial trabeculotomy with microcatheter $(45.5 \pm 25.0 \%$, mean $\pm \mathrm{SD}, P=0.947)$. Segmental outflow has been reported in human eyes, at any given time, only a fraction of the outflow pathways are actively involved in aqueous humor drainage $\mathrm{e}^{21-23}$ and only two aqueous veins could account for all of aqueous outflow in normal human eye. ${ }^{24}$ We then can speculate that the circumferential angle has no need to be opened to $360^{\circ}$ to maintain the normal IOP; a partial of $120^{\circ}$ or more may be enough for outflow drainage, as approved by the successfully IOP control of the traditional $120^{\circ}$ trabeculotomy when the surgery correctly cut open $120^{\circ}$ angle. However, limited by our small sample size, this warrants further investigation.

The available surgical repertoire for PCG has remained relatively unchanged for many years with most progress owing to modifications of the existing surgeries. A major advance has been the concept of incising the whole angle (circumferential trabeculotomy) with either suture ${ }^{11-14}$ or microcatheter. ${ }^{15,16}$ With surgical destruction of the Schlemm's canal and potential severe angle maldevelopment involving Schlemm's canal, ${ }^{19,25-27}$ the microcatheter could not pass $360^{\circ}$. Therefore, it would be encountered in eyes with previous angle surgeries frequently and even in eyes without previous angle surgeries. In cases without previous angle surgeries in our study, complete catheterization was achieved in 15 of 20 (75.0\%) PCG eyes with the assistance of the illuminated microcatheter. It is consistent with the success rate reported in literature (67 to $75 \%$ ). ${ }^{15,16}$ Circumferential trabeculotomy however may be difficult, if not impossible, in cases with previous angle surgeries owing to surgical destruction of the Schlemm's canal. In the 22 eyes with previous failed angle surgeries, complete catheterization with the microcatheter was achieved in four eyes, indicative of an intact Schlemm's canal despite previous surgical injuries. This may explain why previous traditional trabeculotomy failed, as the trabeculotome may have been inadvertently or inaccurately placed or advanced. ${ }^{28}$ The illuminated tip facilitates the placement and advancement of the microcatheter in Schlemm's canal and improves accuracy, making partial trabeculotomy more effective than traditional rigid probe trabeculotomy. ${ }^{29}$

In this study, the microcatheter-assisted trabeculotomy resulted in no complications other than transient hyphema requiring no intervention in all our patients. With the illuminated tip continuously verifying the catheter location within the Schlemm's canal, the microcatheter improves the safety of $360^{\circ}$ trabeculotomy by preventing inadvertent misdirection and tissue disruption.

Our study is limited by the small sample size and relatively short follow-up duration. And the retrospective nature of this study may need further prospective randomized controlled trials to illustrate this issue better.

In conclusion, a flexible microcatheter with an illuminated, atraumatic tip can be successfully used 
to catheterize Schlemm's canal, and perform partial and full circumferential trabeculotomy for the treatment of PCG. It seems to provide excellent pressure-lowering effect with minimal complications in the early postoperative course (12 months) in PCG with previous angle surgeries, and the effect was comparable to that in eyes that underwent this procedure as an initial intervention. Further research is needed to determine the long-term success of this procedure.

\section{Summary}

What was known before

- The available surgical repertoire for PCG has remained relatively unchanged for many years with most progress owing to modifications of existing surgeries. A major advance has been the concept of incising the whole angle (circumferential trabeculotomy) with either suture or microcatheter. Although cases with previous angle surgeries are typically considered to have poor prognosis, the previous angle surgeries may make these cases even more challenging, owing to the limited area intact for additional surgery and difficulty accessing the inferior region with the trabeculotome.

\section{What this study adds}

- The main advantage of microcatheter-assisted trabeculotomy is the ability to treat the entire angle to the maximum degree with a single incision, especially the inferior angle for eyes with previous angle surgeries, thereby maximizing the IOP-lowering benefit. The illuminated tip facilitates the placement and advancement of the microcatheter in Schlemm's canal and improves accuracy, making partial trabeculotomy more effective than traditional rigid probe trabeculotomy. Microcatheterassisted trabeculotomy represents a reasonable choice of initial and repeat surgical treatment for PCG. Our results will support clinicians to give a reconsideration of using this procedure in cases with previous angle surgeries.

\section{Conflict of interest}

The authors declare no conflict of interest.

\section{Acknowledgements}

We thank Dr Debbie S Kuo of Duke University for her input during the manuscript revision.

\section{References}

1 McPherson SD Jr. Results of external trabeculotomy. Trans Am Ophthalmol Soc 1973; 71: 163-167.

2 Quigley HA. Childhood glaucoma: results with trabeculotomy and study of reversible cupping. Ophthalmology 1982; 89(3): 219-226.
3 Luntz MH. The advantages of trabeculotomy over goniotomy. J Pediatr Ophthalmol Strabismus 1984; 21(4): 150-153.

4 Anderson DR. Trabeculotomy compared to goniotomy for glaucoma in children. Ophthalmology 1983; 90(7): 805-806.

5 Akimoto M, Tanihara H, Negi A, Nagata M. Surgical results of trabeculotomy ab externo for developmental glaucoma. Arch Ophthalmol 1994; 112(12): 1540-1544.

6 Gregersen E, Kessing SV. Congenital glaucoma before and after the introduction of microsurgery. Results of 'macrosurgery' 1943-1963 and of microsurgery (trabeculotomy/ectomy) 1970-1974. Acta Ophthalmol (Copenh) 1977; 55(3): 422-430.

7 Rohen JW, Harms H, Barany E. Discussion on new methods of glaucoma surgery. Bibl Ophthalmol 1970; 81: 154-160.

8 Chang TC, Cavuoto KM. Surgical management in primary congenital glaucoma: four debates. J Ophthalmol 2013; 2013: 612708.

9 Ikeda H, Ishigooka H, Muto T, Tanihara H, Nagata M. Longterm outcome of trabeculotomy for the treatment of developmental glaucoma. Arch Ophthalmol 2004; 122(8): $1122-1128$.

10 Terraciano AJ, Sidoti PA. Management of refractory glaucoma in childhood. Curr Opin Ophthalmol 2002; 13(2): 97-102.

11 Beck AD, Lynch MG. 360 degrees trabeculotomy for primary congenital glaucoma. Arch Ophthalmol 1995; 113(9): $1200-1202$.

12 Neely DE. False passage: a complication of 360 degrees suture trabeculotomy. J AAPOS 2005; 9(4): 396-397.

13 Verner-Cole EA, Ortiz S, Bell NP, Bell NP, Feldman RM. Subretinal suture misdirection during 360 degrees suture trabeculotomy. Am J Ophthalmol 2006; 141(2): 391-392.

14 Beck AD, Lynn MJ, Crandall J, Mobin-Uddin O. Surgical outcomes with 360-degree suture trabeculotomy in poorprognosis primary congenital glaucoma and glaucoma associated with congenital anomalies or cataract surgery. J AAPOS 2011; 15(1): 54-58.

15 Girkin CA, Rhodes L, McGwin G, Marchase N, Cogen MS. Goniotomy versus circumferential trabeculotomy with an illuminated microcatheter in congenital glaucoma. J AAPOS 2012; 16(5): 424-427.

16 Sarkisian SR Jr. An illuminated microcatheter for 360-degree trabeculotomy [corrected] in congenital glaucoma: a retrospective case series. J AAPOS 2010; 14(5): 412-416.

17 Beck A, Chang TCP, Freedman S. Definition, classification and differential diagnosis in childhood glaucoma. In: Weinreb RN, Grajewski A, Papadopoulos M, Grigg J, Freedman S (eds). WGA Consensus Series - 9. Kugler Publications: Amsterdam, 2013, pp 3-10.

18 Shaffer RN. Prognosis of goniotomy in primary infantile glaucoma (trabeculodysgenesis). Trans Am Ophthalmol Soc 1982; 80: 321-325.

19 Walton DS, Katsavounidou G. Newborn primary congenital glaucoma: 2005 update. J Pediatr Ophthalmol Strabismus 2005; 42(6): 333-341.

20 Papadopoulos M, Edmunds B, Fenerty C, Khaw PT. Childhood glaucoma surgery in the 21st century. Eye 2014; 28(8): 931-943.

21 Keller KE, Bradley JM, Vranka JA, Acott TS. Segmental versican expression in the trabecular meshwork and involvement in outflow facility. Invest Ophthalmol Vis Sci 2011; 52: 5049-5057. 
22 Yang CY, Liu Y, Gong H. Effects of Y27632 on aqueous humor outflow facility with changes in hydrodynamic pattern and morphology in human eyes. Invest Ophthalmol Vis Sci 2013; 54: 5859-5870.

23 Hann CR, Fautsch MP. Preferential fluid flow in the human trabecular meshwork near collector channels. Invest Ophthalmol Vis Sci 2009; 50: 1692-1697.

24 Johnstone M, Martin E, Jamil A. Pulsatile flow into the aqueous veins: manifestations in normal and glaucomatous eyes. Exp Eye Res 2011; 92: 318-327.

25 Perry LP, Jakobiec FA, Zakka FR, Walton DS. Newborn primary congenital glaucoma: histopathologic features of the anterior chamber filtration angle. J AAPOS 2012; 16: 565-568.
26 Tawara A, Inomata H. Developmental immaturity of the trabecular meshwork in congenital glaucoma. Am J Ophthalmol 1981; 92: 508-525.

27 Hollander DA, Sarfarazi M, Stoilov I, Wood IS, Fredrick DR, Alvarado JA. Genotype and phenotype correlations in congenital glaucoma: CYP1B1 mutations, goniodysgenesis, and clinical characteristics. Am J Ophthalmol 2006; 142: 993-1004.

28 McPherson Jr SD, McFarland D. External trabeculotomy for developmental glaucoma. Ophthalmology 1980; 87(4): 302-305.

29 Shi Y, Wang H, Yin J, Li M, Zhang X, Xin C et al. Microcatheter-assisted trabeculotomy versus rigid probe trabeculotomy in childhood glaucoma. Br J Ophthalmol 2015; 100(9): 1257-1262. 